\title{
Makerspaces, flexible and inclusive learning environments. A scoping
} review

\author{
Makerspace, ambienti di apprendimento flessibili e inclusivi. Una scoping \\ review
}

\author{
Laura Menichettia, Silvia Michelettab,1 \\ a Università degli Studi di Firenze, laura.menichetti@unifi.it \\ ${ }^{b}$ Università degli Studi di Firenze, silvia.micheletta@unifi.it
}

\begin{abstract}
The contribution analyzes the scientific literature from 2000 to 2021 to determine in which training contexts makerspaces are used and for what purposes, for preschool and school age children and youth. The scoping review shows that many experiences are documented in literature, but few experimental studies exist. However, the examples collected, of a constructionist nature, show some privileged areas of intervention: makerspaces are recognized as a valid tool for the development of problem solving and creativity skills, for a more conscious application in the technical-scientific-artistic disciplines, for the creation of inclusive contexts, in situations of social disease or of people more frequently excluded and underrepresented.
\end{abstract}

Keywords: makerspace; education; school; inclusion; literature review.

\section{Sintesi}

Il contributo analizza la letteratura scientifica dal 2000 al 2021 per determinare in quali contesti formativi siano utilizzati i makerspace e con quali finalità, per bambini e ragazzi di età prescolare e scolare. La scoping review mostra che in letteratura sono documentate molte esperienze, ma esistono pochi studi sperimentali. Gli esempi raccolti, di stampo costruzionista, mostrano però alcune aree privilegiate di intervento: i makerspace sono riconosciuti come valido strumento per lo sviluppo di competenze di problem solving e creatività, per una più consapevole applicazione nelle discipline tecnico-scientificheartistiche, per la realizzazione di contesti inclusivi soprattutto in situazioni di disagio sociale o di persone più frequentemente escluse e sottorappresentate.

Parole chiave: makerspace; formazione; scuola; inclusione; rassegna della letteratura.

\footnotetext{
${ }^{1}$ I paragrafi 1, 2, 5 sono da attribuire a Laura Menichetti, i paragrafi 3, 4 a Silvia Micheletta, il paragrafo 6 è stato scritto in collaborazione.

Si ringraziano la dott. Claudia Cavicchioli per il suo contributo all'analisi e alla categorizzazione della letteratura e la dott. Marta Pellegrini per il confronto sulla metodologia di ricerca.

L'articolo è frutto di un'attività di ricerca nell'ambito del progetto STEAM2GO - Mobile for promoting maker education in schools democratizing STEAM education and innovation development for all learners (Erasmus+ 2020-1-PL01-KA201-081698), che vede tra i partner l'Università di Firenze, con il coordinamento della prof. Menichetti $(\mathrm{PhD})$ e la partecipazione della dott. Claudia Cavicchioli (PhD) e della dott. Silvia Micheletta (PhD).
} 


\section{I makerspace}

I makerspace sono spazi fisici in cui le persone imparano ad usare strumenti, sperimentano le caratteristiche di materiali e applicazioni, intraprendono percorsi di progettazione, si misurano in attività manuali e partecipano a comunità, per dare luogo a soluzioni innovative e dare spazio alla propria creatività, realizzando artefatti unici in qualità di novelli artigiani.

Questo fai-da te (do-it-yourself, DIY) si avvale dell'uso del web e di applicazioni in rete, si sviluppa solitamente grazie a tecnologie digitali (microprocessori, sensori, stampanti 3D, macchine a controllo numerico, software $\mathrm{CAD}$, etc.), prevede l'uso di attrezzi più tradizionali (utensili da falegname, strumenti di misura, kit di cucito, etc.), e valorizza materiali poveri di uso comune (stoffa, legno, scatole di cartone, oggetti di plastica, etc.) $\mathrm{Al}$ di là di questa connotazione che combina fisicità e virtualità, però, già nel $2008 \mathrm{i}$ futurologi di Palo Alto avevano messo in luce l'impronta sociale che avrebbe guidato l'agire dei maker e sottolineato la rilevanza del loro operato: "due forze future, una prevalentemente sociale, una prevalentemente tecnologica, si intersecano per trasformare il modo in cui beni, servizi ed esperienze [...] saranno progettati, prodotti e distribuiti. [...] [I maker, $n d a$ ], ispirati dalla diffusione degli ideali open source, si aspettano di poter personalizzare i prodotti che acquistano. E ciò che non possono acquistare, lo costruiscono da zero" (Pescovitz, 2008, p. 1).

Il Maker Movement affonda le proprie radici nella controcultura degli anni Sessanta e Settanta, dà dignità al riuso, alla riparazione, all'assemblaggio creativo, ponendosi agli antipodi del dilagante consumismo degli anni Ottanta e Novanta, e pratica una contaminazione tra bricolage, arte, invenzione, gioco (Honey \& Kanter, 2013). Nel 2005 Dale Dougherty, per indicare questi artigiani digitali, coniò il termine maker e fondò la rivista Make (Dougherty, 2012), che resta un punto di riferimento per il movimento. Esso è costituito da comunità di persone orientate a fare (make), condividere (share), donare (give), apprendere (learn), attrezzarsi (tool up), giocare (play), partecipare (participate), supportare (support), cambiare (change), secondo quanto scriverà poi Hatch nel Manifesto del Movimento (Hatch, 2013; Kalish, 2010).

La dimensione sociale del making si realizza e si manifesta oggi grazie all'accesso facilitato a tecnologie open e con la condivisione di spazi fisici anche pubblici in cui dare vita alle comunità e ai prodotti ${ }^{2}$, per la dimensione inclusiva che caratterizza larga parte delle iniziative e per lo sviluppo di comportamenti prosociali (Doctorow, 2009; Sennett, 2009), per il diffondersi di una cultura di consumo consapevole e per la crescente attenzione al processo di valutazione della qualità (Frauenfelder, 2010).

Il verbo forse più usato per descrivere l'azione dei maker è to hack, che significa ad un tempo incidere, farcela, riuscire, ma anche colpire, spaccare, fare a pezzi. Nonostante la due diverse accezioni del verbo, una con connotazione tipicamente positiva e l'altra con connotazione negativa, infatti, nei maker alberga la convinzione che "we could hack the world around us"3 (Dougherty, 2016, p. 14). La rapida evoluzione delle tecnologie, le crisi economiche che hanno caratterizzato gli ultimi decenni, il ripensamento dei sistemi produttivi hanno portato alla riscoperta del lavoro artigiano nelle società avanzate, in forme

2 Tra i numerosi riferimenti, nel panorama internazionale spicca Fab Foundation (https://fabfoundation.org/) e nel panorama italiano merita di essere citato Make in Italy (http://www.makeinitaly.org/).

${ }^{3}$ Tradotto letteralmente: "possiamo incidere/cambiare/spaccare il mondo intorno a noi" (trad. a cura delle autrici). 
rinnovate, coerenti con il loro connotarsi come società della conoscenza, generando un'innovazione che parte dal basso (Anderson, 2012), frutto dell'ingegno e della creatività di piccoli gruppi in rete tra loro, replicando un modello di costruzione condivisa di conoscenza già praticata comunemente attraverso il web, secondo quello che appare un processo di democratizzazione dell'innovazione (von Hippel, 2005).

I makerspace possono diversificarsi molto per collocazione, layout, attrezzature, nonché per finalità, prodotti realizzati, risultati conseguiti (Schön, Ebner \& Kumar, 2014). Ad esempio, i prodotti a cui danno vita possono rispondere ai bisogni di nicchie di mercato o contribuire allo sviluppo della piccola e media impresa (Van Holm, 2015): in questi casi i makerspace sono solitamente radicati nella realtà produttiva della zona geografica in cui sorgono e possono essere promossi da enti locali.

I makerspace sono diffusi non solo all'interno della scuola, ma anche all'esterno - nelle biblioteche pubbliche o private, nelle ludoteche, negli spazi ricreativi o nei musei (Halverson \& Sheridan, 2014), riempiono il tempo di bambini e ragazzi che li frequentano con entusiasmo. Negli ultimi anni i makerspace sono stati annoverati in una moltitudine di scritti, ma ancora poco si sa del loro portato pedagogico e del loro uso non meramente ricreativo ma formativo. Esistono studi in proposito? Quali aspetti potrebbero essere da approfondire, quali da indirizzare?

Il presente lavoro si interessa alla dimensione pedagogica che si attua con la proposta di attività formative che prevedono la costruzione, l'uso e la mediazione dei makerspace (European Schoolnet, 2017; Johnson et al., 2016).

\section{Il disegno della ricerca}

\subsection{II metodo}

Il metodo di ricerca utilizzato è quello della scoping review (Arksey \& O'Malley, 2005; Levac, Colquhoun \& O’Brien, 2010; Peters, Godfrey, Khalil, McInerney, Parker, \& Soares, 2015). Tale metodo può essere utilizzato per mappare un'area di ricerca ampia, consentendo, da un lato, di delineare la natura e la dimensione della conoscenza scientifica sul focus di ricerca, verificando non solo l'estensione della ricerca disponibile su un argomento ma anche il modo in cui tale ricerca è stata condotta, e, dall'altro, di cogliere i punti di forza e le criticità, ovvero i gap su cui concentrare eventualmente ulteriori ricerche. Le scoping review sono condotte definendo a priori la pertinenza dei risultati con particolare riguardo a tempo, luogo (ad es. Paese o contesto), fonte (ad es. letteratura peerreviewed o grigia) e origine dei documenti presi in esame (ad es. dalle discipline sanitarie, dall'ambito accademico, etc.).

Come tutti i tipi di revisione, anche la scoping review deve essere condotta in maniera sistematica, trasparente e replicabile.

L'applicazione del metodo prevede un protocollo che può essere sintetizzato nei passi seguenti:

1. individuazione di una query e di criteri di inclusione ed esclusione, secondo cui vagliare un insieme di database di settore per rintracciare la letteratura scientifica appropriata;

2. eliminazione di eventuali record duplicati; 
3. analisi di pertinenza dei contributi, in modo da eliminare tutti quei lavori che, pur rispondendo formalmente alla query, non corrispondono alla ricerca di interesse. Solitamente questo passaggio si svolge in due fasi, procedendo nella prima solo alla lettura di titolo ed abstract e nella seconda alla lettura dell'intero articolo;

4. analisi e categorizzazione delle informazioni raccolte;

5. discussione delle informazioni raccolte ed eventuale proposta di ricerche ulteriori. I passaggi sono sempre frutto del lavoro di più ricercatori che operano separatamente, in modo indipendente e parallelo, per poi confrontarsi ed eventualmente discutere i risultati conseguiti facendosi supportare, laddove se ne ravvisi la necessità, anche da ulteriori esperti coinvolti su segmenti specifici della procedura.

\subsection{Le domande di ricerca}

Le domande a cui la presente ricerca vuole dare risposta sono tre:

1. in quali contesti educativi sono utilizzati i makerspace con bambini e ragazzi in età prescolare e scolare?

2. con quali finalità e secondo quali modalità formative tali makerspace sono utilizzati?

3. quali sono gli effetti rilevati a seguito dell'uso dei makerspace nei contesti sopra indicati?

Si noti che si è definita l'età degli alunni e degli studenti, ma si accolgono studi in contesti formali (scuola) e non formali (biblioteche, ludoteche, spazi ricreativi, musei, etc.).

Gli effetti possono essere rilevati con studi qualitativi/quantitativi/misti, esperienze/studi sperimentali, osservazioni sistematiche, ma anche studi qualitativi, studi di caso e interviste, volti a ricercare, cogliere e analizzare le percezioni dei partecipanti coinvolti nell'uso dei makerspace, sia che essi siano i soggetti in formazione, sia che essi siano gli educatori o formatori.

\section{La strategia di ricerca}

\subsection{La query}

L'indagine della letteratura è stata condotta online, dando seguito alle domande di ricerca sopra illustrate. Si è proceduto quindi a formulare la query bilanciando - attraverso gli operatori booleani - le necessità di essere sufficientemente selettivi e di non perdere risultati pertinenti. Ne è risultata una domanda costituita da due criteri in congiunzione tra loro:

- il primo si riferisce alla individuazione dell'oggetto di ricerca, in questo caso i makerspace;

- il secondo restringe la ricerca al contesto d'uso di interesse, cioè il settore education.

Per raccogliere tutti i documenti pertinenti si è provveduto quindi a identificare sinonimi ed espressioni alternative rispetto agli iniziali makerspace e education, e sono state indicate tutte le diverse forme grammaticali che corrispondono a singolari/plurali, coniugazioni, forme derivate. 
$\mathrm{Nel}$ suo complesso la query risultante è stata la seguente: (makerspace* OR fablab* OR "maker space*" OR "maker movement" OR hackerspace* OR hackspace* OR hacklab* OR “creative space*” OR tinker*) AND (education* OR learn* OR teach* OR instruction* OR pedagog*).

La ricerca è stata condotta in alcuni repository specializzati per il settore education:

- Education Source (https://www.ebsco.com/it-it), che indicizza circa 3500 riviste di livello accademico, 3300 atti di convegni, 530 monografie, integrato da APA PsycInfo, che a sua volta indicizza circa 2500 riviste accademiche, oltre a libri e tesi;

- Scopus (https://www.scopus.com/home.uri), che indicizza più di 23700 riviste e oltre 194000 libri;

- JSTOR (https://www.jstor.org/) che indicizza oltre 26000 riviste accademiche e circa 90000 libri;

Ognuno di questi repository prevede proprie modalità di ricerca e per questo è stato tecnicamente necessario adattare la query di base, ovviamente senza modificarne in alcun modo il significato. Nello specifico, poiché JSTOR non accetta stringhe di ricerca più lunghe di 200 caratteri, è stato necessario segmentare la query formulandola in due passi, salvo riaggregare poi i risultati.

Tutte le ricerche sono state condotte, tramite l'opzione "ricerca avanzata", all'interno del campo abstract per poter cogliere in maniera pertinente e precisa le risorse disponibili.

\subsection{Criteri di inclusione e criteri di selezione degli articoli}

All'interno dei repository considerati, gli articoli ritenuti di interesse sono stati quelli corrispondenti ai seguenti criteri di ricerca:

- accesso: articoli pubblicati in modalità open access, oppure raggiungibili attraverso il sistema bibliotecario dell'Università degli Studi di Firenze ${ }^{4}$;

- tipologia di studio: articoli teorici, esperienze, studi sperimentali, studi primari e secondari, studi condotti secondo metodi qualitativi, quantitativi e misti;

- partecipanti: bambini e ragazzi in età prescolare e scolare (escluse quindi le università o la formazione degli adulti). Nessuna limitazione per genere, etnia, numerosità del campione $\mathrm{o}$ altro;

- setting: contesti formali (scuole private o pubbliche di ogni ordine e grado) e non formali (biblioteche private o pubbliche, ludoteche, spazi ricreativi, musei, etc.);

- geografia: tutti i Paesi;

- risultati: nessuna limitazione per quanto riguarda i risultati attesi, studiati, rilevati. Sono stati considerati studi orientati agli apprendimenti, alle strategie didattiche, alla partecipazione, alla motivazione, etc. Sono stati considerati articoli relativi sia a risultati misurati, sia a percezioni di risultato;

- orizzonte temporale: studi dal 2000 al 2021;

- lingua: articoli in inglese, italiano, francese, spagnolo;

- tipo di pubblicazione: articoli in riviste scientifiche e con revisione tra pari.

\footnotetext{
${ }^{4}$ L'Università mette a disposizione abbonamenti a più di 80000 riviste scientifiche, consentendo la lettura del testo completo degli articoli in esse pubblicati.
} 
I criteri di inclusione sono stati applicati con modalità tecniche diverse a seconda dei repository interrogati, ma sempre rispettando la totalità delle scelte effettuate.

I metadati dei contributi selezionati sono stati importati in Zotero e si è proceduto alla eliminazione automatizzata dei duplicati. I metadati degli articoli rimasti sono stati successivamente importati in Excel e si è proceduto ad una seconda fase, questa volta semiautomatica, di eliminazione dei restanti contributi duplicati (la ricerca di Zotero talvolta fallisce per minime differenze di trascrizione nel titolo o nel nome dell'autore).

A questo punto ha preso l'avvio il processo di selezione volto a identificare gli articoli rilevanti: tale selezione è stata condotta in modo indipendente dalle due autrici del presente contributo, in due diverse fasi. Durante la prima fase, le ricercatrici si sono limitate a leggere il titolo e l'abstract di ciascun articolo e, sulla base di queste informazioni, a decidere se scartare la risorsa perché non pertinente rispetto ai criteri di inclusione stabiliti a priori oppure conservarla per la fase successiva. Durante la seconda fase le ricercatrici hanno letto l'intero testo degli articoli rimasti e hanno proceduto alla categorizzazione di ogni singola risorsa sulla base dei criteri che sono discussi nel paragrafo 5. In entrambi i casi il motivo di eventuale esclusione è stato esplicitato, per rendere il processo replicabile. In caso di disaccordo, le ricercatrici ne hanno discusso con una terza persona esperta.

\section{Risultati della ricerca}

Il risultato del processo di ricerca è illustrato nello schema di Figura 1, realizzato secondo il processo Prisma (Page et al., 2021).

Come primo passo si è proceduto alla identificazione dei risultati estraendoli dai repository di settore scelti:

- si è applicata la ricerca in Education Source (+APA PsycInfo)

- il numero dei risultati ottenuti direttamente dalla query è 1072;

- il numero dei risultati dopo l'applicazione dei criteri di inclusione (attraverso i filtri) è 571;

- si è applicata la ricerca in Scopus

- il numero dei risultati ottenuti direttamente dalla query è 1441;

- il numero dei risultati dopo l'applicazione dei criteri di inclusione (attraverso i filtri) è 540;

- si è applicata la ricerca in JSTOR

- il numero dei risultati ottenuti dalla query è $(46+4)+(23+2)=75$.

Come già indicato, in questo caso la query è stata segmentata in due fasi perché il campo di ricerca non accetta più di 200 caratteri.

Inoltre, seguendo le avvertenze del repository, la ricerca è stata effettuata non solo nel campo "Abstract", ma anche nel campo "Titolo", poiché solo il 10\% degli articoli di questo repository è corredato di metadati nel campo "Abstract";

- il numero dei risultati dopo l'applicazione dei criteri di inclusione (attraverso i filtri) è $(34+4)+(10+2)=50$;

per effetto dell'estrazione dai repository si sono quindi ottenuti in totale 1161 contributi scientifici. 
Open Journal per la formazione in rete

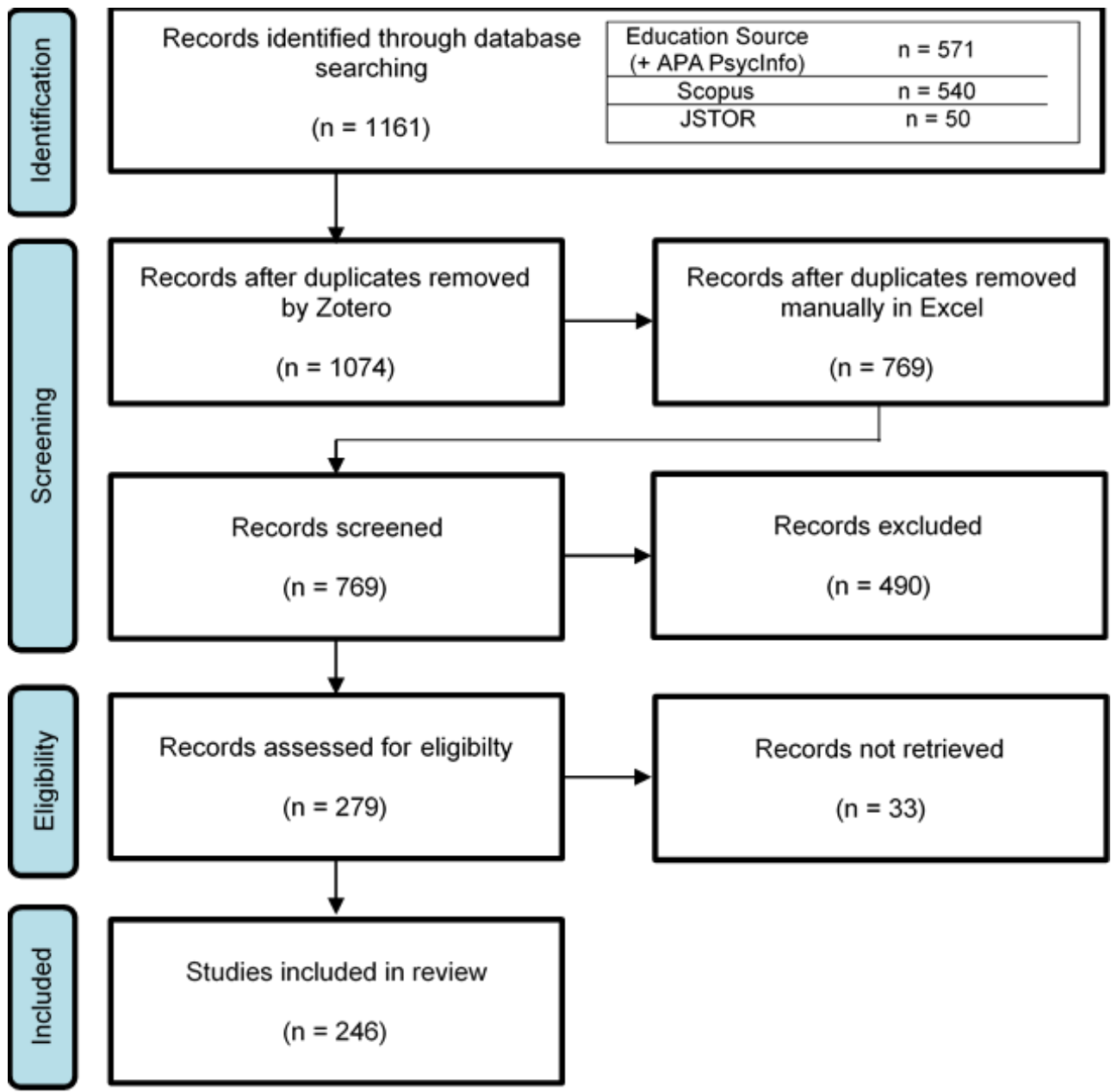

Figura 1. Diagramma PRISMA relativo al processo di selezione dei risultati.

Dopo l'eliminazione dei duplicati (effettuata prima automaticamente con Zotero e poi in maniera semiautomatica con Excel) si sono ottenuti 769 contributi, così distribuiti:

- $\quad$ Education Source (+APA PsycInfo) 474

- Scopus

- JSTOR

La fase successiva (screening 1) ha previsto la lettura di titolo e abstract di ciascun contributo, per eliminare quelli non pertinenti l'applicazione dei criteri di inclusione precedentemente definiti. Questa attività ha integrato quella selezione che i filtri dei repository non consentono di svolgere in maniera automatica. I contributi rimasti sono quindi risultati 279, così distribuiti:

- Education Source (+APA PsycInfo)

189

- Scopus

- JSTOR 5

I file sono stati quindi scaricati in locale, su personal computer, per l'analisi di dettaglio. $\mathrm{Nel}$ compiere questa azione, nonostante la richiesta e l'apparente disponibilità di tutti i 
lavori in open access o nel database dell'Università, è emerso che 33 contributi non risultavano accessibili. I contributi da esaminare quindi sono risultati 246 , così distribuiti:

- Education Source (+APA PsycInfo)

- Scopus

- JSTOR

\section{Categorizzazione dei risultati e loro discussione}

L'analisi dei 246 contributi ha previsto la loro lettura integrale da parte delle due ricercatrici: esse hanno agito in maniera indipendente, sulla base di uno schema deciso concordemente a priori e collaudato con una serie di esempi svolti congiuntamente per assicurare una maggiore uniformità di comportamento; le ricercatrici si sono quindi confrontate a posteriori sui casi non parimenti considerati, chiamando in causa in tali situazioni anche un soggetto terzo, che ha fornito di volta in volta la sua valutazione altrettanto indipendente.

Si è proceduto ad una categorizzazione che fosse in linea con le domande di ricerca.

La scelta delle categorie ha subito un processo iterativo di definizione: le categorie, infatti, erano in larga parte predeterminabili ma la lettura dei contributi ne ha suggerito un affinamento in itinere. Tale adeguamento è esattamente ciò che ci si aspettava a fronte di una scoping review, che, a differenza di altri tipi di revisione della letteratura, è particolarmente indicata nelle situazioni in cui il dominio di ricerca sia ancora non sufficientemente esplorato e studiato nelle sue dimensioni costitutive.

\subsection{Individuazione dei lavori pertinenti}

La lettura dei contributi nella loro interezza ha portato alla esclusione di alcuni ulteriori articoli (screening 2) che formalmente e dalla sola lettura dell'abstract erano risultati potenzialmente congruenti con $\mathrm{i}$ criteri di inclusione, ma che ad un esame più approfondito non sono risultati in linea con l'analisi dichiarata.

La selezione, infatti, fin dall'inizio è stata svolta procedendo secondo criteri cautelativi, che permettessero di conservare il contenuto informativo più ampio possibile, quindi durante il primo screening sono stati conservati tutti quei contributi in cui alcune informazioni non erano presenti nell'abstract: ad esempio si sono potuti escludere in quella fase i contributi in cui si trattavano makerspace utilizzati da studenti universitari, ma sono stati tenuti quelli che nell'abstract non esplicitavano le età dei partecipanti.

Solo dalla lettura attenta dei testi integrali si è potuto risalire a tutti gli elementi necessari per consentire la determinazione dell'effettiva pertinenza dei lavori, ricavando la seguente tabella:

- articoli esclusi per argomento (focus diverso da makerspace)

- articoli esclusi per partecipanti (età non entro la fascia scolare)

- $\quad$ articoli esclusi per tipologia di pubblicazione (proceedings, ...)

TOTALE esclusi

Sono rimasti quindi 168 contributi del tutto pertinenti con lo studio definito, e quindi 168 contributi da classificare. 


\subsection{La letteratura disponibile}

Secondo quanto tipicamente previsto da una scoping review, la prima indagine ha inteso mettere a fuoco la letteratura disponibile, per comprendere la natura e l'estensione della ricerca svolta negli anni passati.

Il $51 \%$ degli articoli esaminati si riferisce alla realtà statunitense: è negli USA che i makerspace sembrano avere il massimo successo e anche raccogliere le attenzioni della comunità scientifica per il loro studio. Per quanto riguarda le altre realtà, il 19\% degli articoli si riferisce all'Europa, il 9\% all'Asia, 1'8\% all'Australia; 1'8\% al Canada, salvo altre situazioni di minore entità (Figura 2).

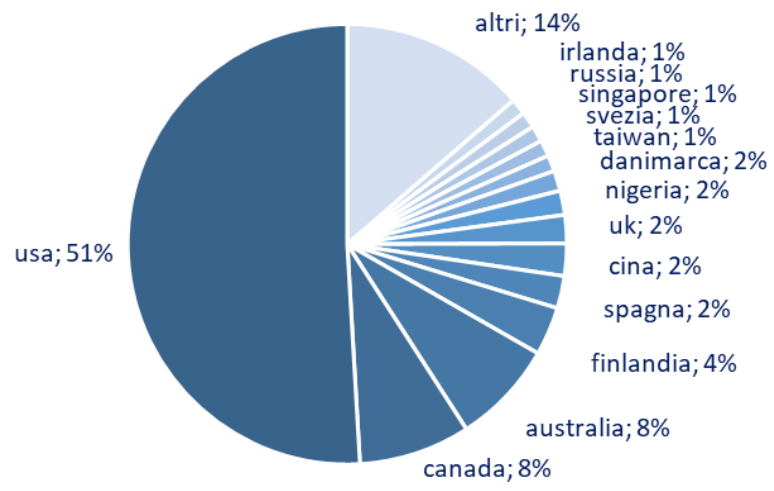

Figura 2. Distribuzione percentuale degli studi per Paese, rispetto al totale esaminato.

L'andamento nel tempo delle pubblicazioni che sono rientrate nello studio è decisamente crescente (Figura 3), con un solo articolo nel 2008 e 42 nel 2020. È significativo osservare come il numero delle pubblicazioni quasi raddoppi nell'arco di due anni tra il 2016 e il 2018 e poi ancora tra il 2018 e il 2020 (il 2021 è ancora in corso).

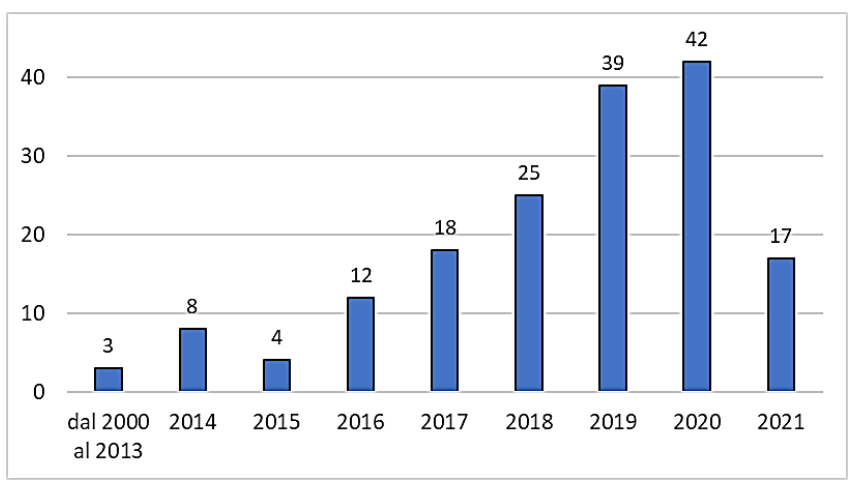

Figura 3. Numero di articoli per anno,

tra quelli ritenuti pertinenti rispetto ai criteri definiti per la scoping review.

Il 20\% degli articoli è di natura teorica: in larga parte sono delle overview della letteratura esistente, sempre di orientamento costruzionista, che però spesso non esplicitano un protocollo specifico di ricerca; in altri casi sono riflessioni e collegamenti alle teorie dell'apprendimento; talvolta suggerimenti per la progettazione di nuovi makerspace. Circa il $24 \%$ dei contributi riporta delle esperienze, dichiarandole come tali: questi lavori intendono porsi come suggerimenti per altri colleghi in modo da contribuire all'attivazione di ulteriori makerspace, oppure sono articoli che terminano raccogliendo le impressioni di 
chi ha condotto l'azione, pressoché sempre molto positive e scritte anche con una certa enfasi. Circa il $46 \%$ dichiara di aver svolto uno studio con un metodo qualitativo, 1 ' $8 \%$ con un metodo quantitativo, il $2 \%$ con un metodo misto.

Raramente, però, sono riportati tutti i passaggi degli studi: molti lavori si limitano a dichiarare che vi sono state delle interviste o sono state condotte delle osservazioni. In relazione a questo fattore di analisi si individuano due approfondimenti da attuare in occasione di una prossima revisione della letteratura:

- occorre distinguere gli studi che solo dichiarano la modalità di intervento e di raccolta dati da quelli che esplicitano i singoli passaggi e quindi forniscono tutti gli elementi per attestare la scientificità dei processi;

- occorre distinguere tra la classificazione operata dagli autori e quella che si può rilevare dal testo dell'articolo. Ad esempio, per quanto possa sempre trattarsi di analisi qualitativa, i risultati di un questionario a risposta aperta sul grado di accettazione del makerspace devono essere considerati diversamente da un'osservazione dei comportamenti dei partecipanti (magari associata allo studio quantitativo su tempi e frequenze di utilizzo), oppure dall'analisi dei commenti registrati durante le attività, etc.

\subsection{I makerspace rappresentati}

La seconda indagine ha riguardato più propriamente l'oggetto della ricerca: la struttura dei makerspace, che sono stati osservati seguendo le domande di ricerca indicate al paragrafo 2.2 .

Nessuno degli articoli selezionati prende in considerazione makerspace in età prescolare, mentre molti si riferiscono a makerspace utilizzati da bambini e ragazzi di età diverse ${ }^{5}$, anche aperti all'intero ciclo scolastico (Figura 4).

\begin{tabular}{|l|c|}
\hline Età & Numero di articoli \\
\hline 3-5 anni (livello scuola dell'infanzia) & 10 \\
\hline 6-8 anni (livello scuola primaria - primo ciclo) & 91 \\
\hline 9-10 anni (livello scuola primaria - secondo ciclo) & 96 \\
\hline 11-13 anni (livello scuola secondaria di primo grado) & 105 \\
\hline 14-16 anni (livello scuola secondaria di secondo grado - biennio) & 98 \\
\hline 17-19 anni (livello scuola secondaria di secondo grado - triennio) & 90 \\
\hline
\end{tabular}

Figura 4. Numero di articoli per fascia di età, tra quelli considerati nella scoping review.

Per quanto riguarda il genere, circa il 10\% dei makerspace si attiva con programmi destinati al genere femminile, circa il $40 \%$ si rivolge indifferentemente a femmine e a maschi, oltre il $50 \%$ non cita il genere come aspetto di attenzione in fase di progettazione e realizzazione della ricerca. I programmi rivolti al genere femminile specificano di voler contribuire

\footnotetext{
${ }^{5}$ Considerare le età a cui l'articolo si riferisce determina anche un numero totale di occorrenze che apparentemente supera il 100\% dei contributi disponibili, proprio perché un articolo può trattare ad esempio di makerspace aperti a ragazzi del biennio e anche del triennio della scuola secondaria di secondo grado, e così per altri ordini e gradi.
} 
all'empowerment di un insieme della popolazione sottorappresentato nelle STEM (Science, Technology, Engineering, Mathematics) o nelle STEAM (Science, Technology, Engineering, Arts, Mathematics).

Oltre il 50\% dei contributi non specifica se il makerspace sia utilizzato da soggetti singoli o da gruppi, lasciando intendere che entrambe le modalità siano praticate o che vi possa essere una flessibilità nell'utilizzo dello spazio creativo. Il 37\%, invece, dichiara esplicitamente che l'attività proposta con il makerspace è da svolgere preferibilmente in gruppo, tramite il confronto e la collaborazione con i pari nello spazio stesso dell'azione. Il restante $10 \%$ prevede, infine, un utilizzo individuale del makerspace, salvo poi ricondursi a comunità di pratica per commenti e condivisioni.

Le finalità con cui i makerspace sono utilizzati risultano molto difficili da determinare $\mathrm{e}$ anche questa rappresenta una direzione ulteriore secondo cui ampliare la ricerca. Tutti i contributi analizzati parlano di STEM e di una dimensione laboratoriale, attiva (spesso definita diversa dal consueto) nell'affrontare le discipline tecnico-scientifiche. Molto più interessante è un indirizzo specificatamente inclusivo che il makerspace in molti casi assume: in un terzo dei casi si è consapevoli del fatto che, per quanto l'obiettivo disciplinare più immediato siano le STEM, la finalità ultima è quella di rivolgersi a fasce della popolazione in situazione di disagio socio-economico o socio-culturale, a genere e etnie sottorappresentate nel campo delle STEM. Non solo in vista di azioni positive a favore di un bilanciamento tra maschi e femmine nelle professioni scientifiche e tecnologiche, ma anche con l'obiettivo di sostenere la partecipazione alle STEM da parte delle minoranze etniche di certe comunità che difficilmente hanno accesso alle tecnologie, contrastando quel digital divide che si manifesta come disparità tra il livello di utilizzo delle tecnologie e la consapevolezza della necessità di un approccio scientifico sistematico. I makerspace contribuiscono alla creazione di un'identità nelle STEM, alla consapevolezza di poter partecipare, all'empowerment di coloro che la cultura diffusa pone ai margini, alla disgregazione degli stereotipi di settore che passano attraverso le narrazioni (verbali o visive) e si consolidano fin dalla più tenera età.

Una delle maggiori criticità, invece, risiede nel fatto che circa il $60 \%$ dei contributi non accenna al tema della valutazione dei risultati, oppure nel contributo si dichiara che vi è stata una valutazione ma si comprende che si tratti solo di un'indagine di gradimento. Nel $23 \%$ dei casi si raccolgono le percezioni di insegnanti e studenti. Soltanto nel $17 \%$ dei casi si procede con una valutazione di risultato, e in questi casi le valutazioni risultano positive. Sono ancora pochi però gli studi sperimentali nel settore, spesso gli articoli sono frutto degli stessi partecipanti all'intervento, risultando quindi in parte condizionati dal coinvolgimento nell'azione.

L'acronimo STEM- e a maggior ragione quello STEAM, introducendo le Arti - secondo alcuni rimanda ad un insieme non del tutto coerente di concetti, conoscenze, percorsi. Non sempre per gli esperti di una delle aree comprese nelle STEAM le altre assumono rilevanza, e i portatori di interessi spesso appaiono diversi, cosicché a livello epistemologico vi è solo un parziale riconoscimento reciproco (Breiner, Harkness, Johnson \& Koehler, 2012). Il Maker Movement ha il pregio di superare le antinomie tra scienza pura e scienza applicata (Dewey, 1938), tra scienza e tecnologia, tra arte e artigianato, e funge da catalizzatore per una visione olistica che va oltre le singole discipline (Bullock \& Sator, 2015). La varietà dei contesti rappresentati documenta la flessibilità dello strumento.

Nelle scuole i docenti propongono i makerspace come una nuova strategia che consente di conseguire competenze disciplinari e di sviluppare competenze trasversali. Alcuni 
makerspace sono attivati specificatamente per imparare a collaborare, a confrontarsi, ad esercitare la propria leadership.

Attraverso la lettura dei contributi si apprende che nei makerspace si sviluppano le competenze di problem solving che potremmo dire di tipo analitico, quelle che si appoggiano sui costrutti del pensiero logico-razionale. Si deve a Dewey (1910) l'esplicitazione chiara di un modello ricorrente di pensiero che procede dalla rilevazione del problema alla sua soluzione: "five logically distinct steps, (i) a felt difficulty; (ii) its location and definition; (iii) suggestion of possible solution; (iv) development by reasoning of the bearings of the suggestion; (v) further observation and experiment leading to its acceptance or rejection; that is, the conclusion of belief or disbelief" ${ }^{\prime 6}(\mathrm{p} .72)$. Il feedback intrinseco nel comportamento dei prodotti realizzati nei makerspace e la necessaria gestione dell'errore supportano la strutturazione e ristrutturazione dei problemi.

Come sappiamo, però, un'ampia gamma di situazioni problematiche può trarre beneficio dalla messa in atto di comportamenti creativi (Sternberg, 1999; Ward, 2012), che seguono metodi non convenzionali, fino a illuminazioni (insight, eureka effect, aha! moment) e i makerspace possono raccordare queste due dimensioni logico-razionale e creativa. Le diverse teorie sulla creatività concordano sul fatto che essa non si risolva solo nell'illuminazione, ma preveda fasi di comprensione del problema a monte e di verifica a valle (Wallas, 1926) e, secondo alcuni, anche di conclusiva condivisione (Torrance, 1988). $\mathrm{Si}$ viene configurando quindi una forma di creatività che può essere accompagnata, allenata, formata (Frison \& Menichetti, 2020), nella successione di un pensiero divergente che produce molte ipotesi da punti di vista diversi, anche inusuali (es. attraverso brainstorming), e di un pensiero convergente che mira a ridurre le possibilità, alla ricerca di quella ottimale, attraverso un processo di valutazione e di accettazione (Isaksen \& Treffinger, 2004) che il makerspace può fisicamente concretizzare da un lato nel prodotto realizzato, dall'altro nella costruzione di comunità.

\section{Conclusioni}

La letteratura esaminata è soltanto una parte di quella disponibile: ad esempio non è stata considerata la letteratura grigia, così come esistono altri repository di settore in cui agire: l'applicazione della stessa query sul repository ERIC restituisce circa 20000 risultati, che probabilmente corrisponderanno ad un numero molto inferiore di lavori pertinenti identificati attraverso l'applicazione dei criteri di inclusione scelti, ma che comunque lasciano intravedere la possibilità di condurre una ricerca più ampia.

Ulteriori elementi da approfondire corrispondono a quelle criticità rilevate in questo primo studio e segnalate nel paragrafo precedente.

Nonostante queste limitazioni, il quadro che emerge dalla scoping review consente di rilevare una carenza di studi sperimentali a favore di una rappresentazione di esperienze e

\footnotetext{
${ }^{6}$ Tradotto letteralmente: "cinque passaggi logicamente distinti, (i) una difficoltà sentita; (ii) la sua ubicazione e definizione; (iii) suggerimento di possibile soluzione; (iv) sviluppo attraverso il ragionamento delle conseguenze del suggerimento; (v) ulteriori osservazioni ed esperimenti che portino alla sua accettazione o rifiuto; cioè, la conclusione del credere o non credere" (trad. a cura delle autrici).
} 
di pratiche. Possiamo dire di essere ancora nella fase crescente dell'Hype Cycle (Blosch \& Fenn, 2018), laddove l'entusiasmo sembra sopravanzare la valutazione di reali benefici.

Nei casi meglio documentati sono rappresentati makerspace abbastanza differenziati, proposti in modalità variabili, sempre secondo il modello costruzionista, in contesti formativi - formali (la scuola di ogni ordine e grado) tanto quanto non formali (biblioteche, ludoteche, spazi ricreativi, musei, etc.) - con bambini e ragazzi di età prescolare e scolare con lo scopo di favorire lo sviluppo di competenze di problem solving, imprenditorialità e creatività. Fondamentale l'impiego nel sostenere e stimolare gli apprendimenti nelle discipline STEAM, e nel realizzare contesti inclusivi, soprattutto nelle situazioni di disagio, fino a contribuire alla formazione dell'identità nelle STEAM anche per quelle persone spesso sottorappresentate nei domini specifici (per genere, per etnia, perché appartenenti a minoranze).

\section{Riferimenti bibliografici}

Anderson, C. (2012). Makers: The new industrial revolution. Random House.

Arksey, H., \& O’Malley, L. (2005). Scoping studies: towards a methodological framework. International Journal of Social Research Methodology, 8(1), 19-32. https://doi.org/10.1186/s12916-015-0465-6 (ver. 26.07.2021).

Blosch, M., \& Fenn, J. (2018). Understanding Gartner's Hype Cycles. https://www.gartner.com/en/documents/2018/8/3887767-understanding-gartners-hype-cycles01 (ver. 26.07.2021).

Breiner, J.M., Harkness, S.S., Johnson, C.C., \& Koehler, C.M. (2012). What is STEM? A discussion about conceptions of STEM in education and partnerships. School Science and Mathematics, 112(1), 3-11. https://doi.org/10.1111/j.19498594.2011.00109.x (ver. 26.07.2021).

Bullock, S.M., \& Sator, A.J (2015). Maker pedagogy and science teacher education. Journal of the Canadian Association for Curriculum Studies, 13(1), 60-87.

Dewey, J. (1910). How we think. Lexington, MA: Heath \& Company.

Dewey, J. (1938). Unity of science as a social problem. Chicago, IL: University of Chicago Press.

Doctorow, C. (2009). Makers. New York, NY: HarperCollins.

Dougherty, D. (2012). The Maker Movement. Innovations, 7(3), 11-14.

Dougherty, D. (2016). Free to make: How the maker movement is changing our schools, our jobs, and our minds. Berkley, CA: North Atlantic Books.

Education Source. https://www.ebsco.com/it-it (ver. 26.07.2021).

European Schoolnet (2017). Open book of educational innovation. Brussels. http://www.eun.org/documents/411753/817341/Open_book_of_Innovational_Ed ucation.pdf/c1044658-5793-408c-8acb-a041338111ef (ver. 26.07.2021).

Fab Foundation. https://fabfoundation.org/ (ver. 26.07.2021).

Frauenfelder, M. (2010). Made by hand: searching for meaning in a throwaway world. New York, NY: Portfolio. 
Frison, D., \& Menichetti, L. (2020). Metodi ludici. Tendenze e didattiche innovative 0-11. Lecce: Pensa MultiMedia.

Grant, M.J., \& Booth, A. (2009). A typology of reviews: an analysis of 14 review types and associated methodologies. Health Information and Libraries Journal, 26, 91108. https:// doi.org/10.1111/j.1471-1842.2009.00848.x (ver. 26.07.2021).

Halverson, E.R., \& Sheridan, K. (2014). The maker movement in education. Harvard Educational Review, 84(4), 495-504. https://static1.squarespace.com/static/5bc4e5207fdcb82469b014c6/t/5c0aa9152b 6a281ca2cc3dd5/1544202517946/2014_Halverson\%26Sheridan_HEREssay.pdf (ver. 26.07.2021).

Hatch, M. (2013). The Maker Movement Manifesto: rules for innovation in the new world of crafters, hackers, and tinkerers. New York, NY: McGraw Hill.

Honey, M., \& Kanter, D.E. (2013). Design, make, play: growing the next generation of STEM innovators. New York, NY: Routledge.

Isaksen, S.G., \& Treffinger, D.J. (2004). Celebrating 50 years of reflective practice: versions of creative problem solving. Journal of Creative Behavior, 38, 75-101.

Johnson, L., Becker, S.A., Cummins, M., Estrada, V., Freeman, A., \& Hall, C. (2016). NMC Horizon Report: 2016 higher education edition (pp. 1-50). Austin, TX: The New Media Consortium.

JSTOR. https://www.jstor.org/ (ver. 26.07.2021).

Kalish, J. (21 novembre 2010). DIY "Hackers" tinker everyday things into treasure. $\mathrm{Npr}$. https://www.npr.org/2010/11/12/131268511/diy-hackers-tinker-everyday-thingsinto-treasure?t=1627331168888 (ver. 26.07.2021).

Levac, D., Colquhoun, H., \& O'Brien, K.K. (2010). Scoping studies: advancing the methodology. Implementation Science, 5(69), 1-9. https://doi.org/10.1186/17485908-5-69.pdf (ver. 26.07.2021).

Make in Italy. http://www.makeinitaly.org/ (ver. 26.07.2021).

Page, M.J., McKenzie, J.E., Bossuyt, P.M., Boutron, I., Hoffmann, T.C, Mulrow, C.D., ...Moher, D. (2021). The PRISMA 2020 statement: an updated guideline for reporting systematic reviews. $B M J, 372(71), 1-9$. https://doi.org/10.1136/bmj.n71 (ver. 26.07.2021).

Pescovitz, D. (2008). The future of making: the way the things are made is being remade. Palo Alto, CA: Institute for the future.

Peters, M.D., Godfrey, C.M., Khalil, H., McInerney, P., Parker, D., \& Soares, C.B. (2015). Guidance for conducting systematic scoping reviews. International Journal of Evidence Based Healthcare, 13(3), 141-146. http://doi.org/10.1097/XEB.0000000000000050 (ver. 26.07.2021).

Schön, S., Ebner, M., \& Kumar, S. (2014). The maker movement. Implications of new digital gadgets, fabrication tools and spaces for creative learning and teaching. eLearning Papers. Special edition, 39, 86-100. https://openlib.tugraz.at/download.php?id=56a88d76659bc\&location=browse (ver. 26.07.2021).

Scopus. https://www.scopus.com/home.uri (ver. 26.07.2021). 
Sennett, R. (2009). The craftsman. New Haven, CT: Yale University Press.

Sternberg, R. J. (Ed.) (1999). Handbook of creativity. Cambridge, UK: Cambridge University Press.

Sucharew, H. \& Macaluso, M. (2019). Methods for research evidence synthesis: the scoping review approach. Journal of Hospital Medicine, 7, 416-418. https://doi.org/10.12788/jhm.3248 (ver. 26.07.2021).

Torrance, E.P. (1988). The nature of creativity as manifest in its testing. In R.J. Sternberg (Ed.), The nature of creativity: contemporary psychological perspectives (pp. 4375). Cambridge, MA: Cambridge University Press.

Tricco, A.C., Antony, J., Zarin, W., Strifler, L., Ghassemi, M., Ivory, J., ...Straus, S.E. (2015). A scoping review of rapid review methods. BioMedCentral, 13(224), 115. https://doi.org/10.1186/s12916-015-0465-6 (ver. 26.07.2021).

Van Holm, E.J. (2015). Makerspaces and contributions to entrepreneurship. Procedia: Social and Behavioral Science, 195, 24-31. http://doi.org/10.1016/j.sbspro.2015.06.167 (ver. 26.07.2021).

von Hippel, E. (2005). Democratizing innovation. Cambridge, MA: MIT Press.

Wallas, G. (1926). The art of thought. London, UK: J. Cape.

Ward, T. B. (2012). Problem solving. In M. D. Mumford (Ed.), Handbook of organizational creativity (pp. 169-187). Cambridge, MA: Academic Press.

Ward, T.B., Smith, S.M., \& Vaid, J. (1997). Conceptual structures and processes in creative thought. In T.B. Ward, S.M. Smith, \& J. Vaid (Eds.), Creative thought: an investigation of conceptual structures and processes (pp. 1-27). Washington, DC: American Psychological Association. https://doi.org/10.1037/10227-001 (ver. 26.07.2021). 